\title{
Delincuencia, infancia y alteridad: una propuesta de inteligibilidad*
}

\section{Otherness from Delinquency and Childhood: A Proposal of Intelligibility}

Recibido: 1 de marzo de 2015 | Revisado: 1 de junio de 2015 | Aceptado: 1 de agosto de 2015

\author{
Boris VALDENEGRo EgOzCue ** \\ Universidad de Playa Ancha, Valparaíso, Chile
}

doi:10.11144/Javeriana.up14-4.diap

Para citar este artículo: Valdenegro, B. (2015). Delincuencia, infancia y alteridad: una propuesta de inteligibilidad. Universitas Psychologica, 14(4), 1473-1484. http://dx.doi.org/10.11144/Javeriana. up14-4.diap

* Desarrollado en el contexto de la beca de doctorado nacional, del programa de formación de capital humano avanzado de CONICYT- Chile.

** Licenciado en Psicología Universidad de Valparaíso. Magister en Psicología Social Universidad de Valparaíso. Docente Departamento Disciplinario de Psicología, Facultad de Ciencias Sociales Universidad de Playa Ancha. Correo electrónico: boris. valdenegro@upla.cl

\section{RES UMEN}

Este artículo se sitúa desde la psicología social comunitaria, abordando los procesos de alteridad sobre la relación que se establece en Chile entre infancia y delincuencia. Se enmarca esta discusión desde la noción de dispositivo, en el contexto de las políticas públicas. Los apartados desarrollan la relación entre infancia, delincuencia y alteridad desde distintas perspectivas (políticas públicas, investigación social, dispositivo foucaultiano, historiografía e intervención social), desarrollando una crítica a los procesos de criminalización de la infancia y proponiendo la noción de alter-infancia como posibilidad de comprensión crítica.

Palabras clave

psicología social comunitaria; infancia; delincuencia; alteridad; dispositivo; políticas públicas

\section{A B S T R A C T}

This article is based on communitarian social psychology perspective. It will analyse the process of otherness in the relationship between childhood and delinquency. It will discuss in the context of social policies using the notion of device (dispositif). The following sections will develop the relationship between childhood, delinquency and otherness using several approaches: social policies, research in social sciences, the notion of device (Foucault) and historiography, developing a critical over the criminalization process to childhood, and proposing the idea of Alter- Childhood as critical compehension.

Keywords

social communitarian psychology; childhood; delinquency; otherness; device; social policies 
La relación entre políticas públicas, infancia y delincuencia en Chile se inscribe en un ámbito de acción y estudio que se encuentra saturado de supuestos indiferenciados, reproduciéndose certezas estigmatizantes antes que posiciones críticas. El propósito de este artículo es problematizar, desde la perspectiva de la psicología social comunitaria, la relación entre infancia, delincuencia y alteridad, sobre cuatro objetivos: 1) la política pública y la subjetivación diferencial de infancias; 2) las relaciones entre infancia, delincuencia y alteridad; 3 ) la noción de dispositivo como productor de alteridad en infancia y 4) procesos sociohistóricos que subjetivan infancias desde la alteridad.

A este respecto, la psicología social comunitaria (Alfaro, 2009, 2013; Montero, 2010, 2011) contribuye a desarrollar perspectivas desnaturalizadoras de los procesos sociales, históricos y culturales contenidos en estos ejes reflexivos, desde dimensiones conceptuales, ético-políticas y disciplinares. En lo conceptual, posibilita la comprensión intersubjetiva de la relación entre infancia y delincuencia, desde una perspectiva crítica de las relaciones sociales (Montero, 2011; Piper, 2002), desmitificando su carácter de manifestación objetiva (Montero, 2010; Montero \& Montenegro, 2006). A nivel ético-político, facilita la visibilización de las tensiones entre la perspectiva garantista y proteccional hacia las infancias, aportando al debate sobre los procesos de criminalización en la intervención (Sepúlveda, 2011) y cuestionando las perspectivas estigmatizadoras (Llobet, 2010; Olmos, 2012). A nivel disciplinar, permite definir la intervención desde un carácter situado (Montenegro \& Pujol, 2003), aportando a la complementariedad entre la formulación, diseño e implementación de la intervención social, al considerar las tensiones entre los agentes disciplinares, los formuladores y sujetos intervenidos (Alfaro, 2013), en una lectura compleja de los procesos de intervención (Alfaro, 2009; Piper, 2002). Estas ideas-fuerza serán empleadas para reflexionar acerca de las implicancias de cada uno de los apartados, decantando en la noción de alter-infancia, en tanto proceso de subjetivación de alteridad (Gnecco, 2008).

\section{Políticas públicas e infancias: entre el garantismo y la criminalización}

Las políticas públicas en Chile articulan visiones sobre las infancias, erigiéndose un discurso dominante desde la Doctrina de la Protección Integral (Galvis, 2009; Quiroz, 2011), sustentado normativamente en la Convención de Derechos de la Infancia (Fondo de las Naciones Unidas para la Infancia [UNICEF], 1989), con la pretensión de marcar un hito de tránsito entre la doctrina basada en la noción de menor en situación de vulnerabilidad a otra que concibe a la infancia en tanto sujeto de derechos.

A nivel del diseño de políticas públicas, el accionar del Ministerio de Justicia a través del Servicio Nacional de Menores ha desarrollado la concepción de separación de vías (Werth, 2010), como una expresión de una supuesta diferencia entre la aproximación hacia los denominados adolescentes infractores de ley y los niños y niñas sujetos de promoción de derechos. El devenir de esta perspectiva es cuestionable, siendo muchos de sus efectos y prácticas vulneradoras de derechos humanos (Instituto Nacional de Derechos Humanos [INDH], 2013; UNICEF, 2008).

La política pública orientada hacia los "infractores de ley" se encuentra culturalmente construida desde la noción del miedo al otro (Programa de las Naciones Unidas para el Desarrollo [PNUD], 1998), cimentando la cohesión social en la identificación de éstos como íconos de la peligrosidad e inseguridad (Vergara, 2007), sustentando procesos de criminalización (Sepúlveda, 2011). Es esta radical mirada sobre la infancia criminalizada la que permite reflexionar en torno a la intervención social desde la alteridad.

Si se realiza un análisis a nivel internacional de los programas que intervienen sobre la infancia criminalizada, se destacan las estrategias preventivas centradas en la relación costo-beneficio (Greenwood, 2008), que privilegian la intervención sobre la infancia para disminuir la conducta criminal en la etapa adulta, bajo el supuesto de una mayor permeabilidad de los factores asociados (Jenson, 2010; Mann \& Reynolds, 2006; White, Temple, $\&$ Reynolds, 2010). 
En esta perspectiva, Trentacosta y Shaw (2009) llevaron a cabo un estudio longitudinal sobre la relación entre la autorregulación emocional y el rechazo de pares, observándose una asociación positiva entre este y el comportamiento antisocial, debiéndose trabajar la autorregulación adaptativa tempranamente. Hawkins, Kosterman, Catalano, Hill y Abbot (2008) presentan los efectos de una estrategia de intervención multidimensional en el espacio escolar, reportándose efectos significativos en la reducción de la delincuencia adulta. Mann y Reynolds (2006) desarrollaron una investigación longitudinal sobre la intervención educativa temprana en la delincuencia juvenil, relacionando los factores de intervención y escolarización temprana con la reducción de la delincuencia. Asimismo, Reynolds, Ou y Topitzes (2004) investigaron longitudinalmente los efectos de la participación de preescolares de clase baja en los Centros de Padres e Hijos, evidenciándose mayores logros educativos y menores tasas de arrestos juveniles. Asimismo, Crooks, Scott, Ellis y Wolfe (2011) analizaron un programa de prevención de la violencia escolar, determinándose un efecto amortiguador de este respecto de la comisión de delitos violentos.

Más allá del ámbito escolar, la revisión internacional da cuenta de programas de intervención hacia la infancia que apuntan de modo general a la disminución de riesgos futuros, donde la delincuencia es uno de los factores de riesgo. Geenwood (2008) plantea la importancia de focalizar los esfuerzos en la denominada práctica basada en la evidencia, lo cual implica centrarse en indicadores que delineen buenas prácticas, más allá de la perspectiva conceptual a la base. Cole, Mills, Jenkins y Dale (2005) hicieron una comparación entre programas de intervención temprana sobre la delincuencia, incorporando a la medición determinados indicadores que denominan de desarrollo social, como la satisfacción escolar, la soledad y depresión, concluyendo que no hay diferencias en los resultados entre programas. En ambas experiencias, la intervención temprana es una estrategia consensuada, en la lógica de la posibilidad de incidencia sobre factores asociados (White et al., 2010).
A nivel nacional, se representa el ámbito escolar como factor protector de diversas manifestaciones disfuncionales, definidas dentro de un campo semejante de problemas sociales: consumo de drogas ilícitas, rendimiento escolar, deserción, conductas de riesgo en general y delincuencia, siendo el Servicio Nacional para la Prevención y Rehabilitación del Consumo de Drogas y Alcohol ([SENDA], 2013) el encargado de articular los esfuerzos entre el Ministerio de Educación y el Ministerio del Interior y Seguridad Pública. Fuera del ámbito escolar, los programas se sitúan en la óptica de cumplimiento de penas, en una perspectiva de responsabilización y control de la conducta delictiva (Ministerio de Justicia, 2005).

En síntesis, la perspectiva de factores de riesgo y protectores define la intervención sobre la delincuencia en niños y niñas desde la incidencia de factores contextuales e individuales, consolidando una perspectiva de interacción diádica y de costobeneficio. No se concibe desde allí la posibilidad de comprender la relación entre infancia y delincuencia en tanto manifestación social, histórica y cultural, reduciendo la intervención al ámbito puramente técnico. Se asume así a la infancia como una etapa uniforme, posible y necesaria de intervenir desde la urgencia, antes de llegar a una etapa tardía. Se les concibe como potenciales delincuentes, sintetizándose el negocio de la prevención en "cuidarlos hoy para cuidarnos mañana" (Grupo de trabajo "Niñez", 2006).

En tensión a esta perspectiva, surgen lineamientos críticos agrupables bajo la noción de construcción social de las criminalización. Smith (2009) sostiene una perspectiva interventiva crítica en la esfera judicial, conceptualizando la infancia en tanto construcción social y resaltando su cualidad agencial desde la óptica de los derechos humanos. Complementariamente, Gatti, Tremblay y Vitaro (2009) refieren que la intervención jurídica se centra en las características familiares y sociales, antes que en las condiciones asociadas al delito, afirmando que dicha intervención judicial presenta un efecto iatrogénico, al aumentar la probabilidad de comisión de delitos en la edad adulta. A su vez, Greenwood (2008) sostiene que muchos de los pro- 
gramas considerados en un momento como exitosos incrementan la probabilidad de delincuencia en la etapa adulta. Bernburg, Krohn y Rivera (2006) realizan un estudio longitudinal sobre sujetos infractores de ley, sosteniendo que la intervención de la justicia juvenil acrecienta la probabilidad de involucramiento en la comisión de delitos graves. Desde estos trabajos, se aprecia la pertinencia de entender las políticas públicas como ingenios mecanismos reproductores de criminalización.

\section{Infancia, delincuencia y alteridad: posibilidades desde la investigación social}

La concepción de alteridad da cuenta de procesos subjetivadores inscritos en discursos y prácticas que delimitan la normatividad y la desviación, categorizando a grupos desde relaciones sociales de dominio (Cabruja, 1998). Como heurística científica es multidisciplinar (González, 2009) y se desarrolla desde campos como la antropología, la psicología social, la sociología, la lingüística y la educación, requiriendo una articulación compleja para iluminar los procesos de producción de conocimiento; corresponden a procesos situados sociohistóricamente en la modernidad (Gnecco, 2008), en tensión dialéctica con los procesos de identidad (Flórez, 2007; Heffes, 2012; Rabello, Rocha, Texeira, Alves, \& Antunes, 2006). Desde una perspectiva crítica, los procesos de alteridad cristalizan las representaciones de lo otro sobre distinciones que facilitan la subordinación (Juliano, 1994), desde el ejercicio hegemonizador de su rechazo incluyente, representándolo en una suerte de basurero del Yo (Gnecco, 2008). Lo anterior, resalta el doble juego de la modernidad, en orden a la necesidad de consolidar la figura del Otro monstruoso pero a la vez necesario, centrando el accionar en la administración de la diferencia antes que en su erradicación.

Así entendido, la alteridad se refiere a producciones discursivas que determinan específicas relaciones de poder, las cuales mantienen el orden social y definen vinculaciones de dominio y de subordinación. Se desconocen así las relaciones sociales desde su carácter histórico, produciéndose esencializaciones excluyentes relativamente rígidas, sustentadas en una lectura binarista de las relaciones sociales (Castro-Gómez, 2000).

A partir de lo anterior, la construcción de una infancia criminalizada en oposición a la infancia normalizada (Llobet, 2010) es una cuestión de administración de la delincuencia, no siendo posible ni deseable su erradicación al ser necesaria para la función de normalización social. Se hace necesario conjuntamente el ejercicio de la violencia, dado el carácter violento de la constitución del sujeto moderno en el horizonte colonial y postcolonial (Castro-Gómez, 2000; Gnecco, 2008; Herrera, 2006; Recasens, 2006).

Desde una lectura contemporánea, las lógicas de exclusión binaria se tensionan con los procesos de la modernidad tardía, desde los cuales se asiste a la ruptura de los modos dicotómicos de producir sujetos (Castro-Gómez, 2000), valorándose la diversidad cultural como modo de viabilizar los nuevos modelos de desarrollo (Soria, 2012), desdibujando a su vez la monstruosidad encarnada en la figura de la alteridad (Blaney, 2008; Foucault, 2011). No obstante, el estado del arte muestra una multiplicidad de figuras de exclusión: en torno a dispositivos de sexualidad (Piola, 2008) o los dispositivos jurídico-culturales (Juliano, 1994); desde los fenómenos migratorios (Rea, 2006; Olmos, 2012) o en los dispositivos educativos (Cabruja, 1998; Colmenares, 2004).

Complementariamente, se consolida en la espacialidad de la urbe en tanto lugar de confrontación con el otro, concibiendo la ciudad como el espacio de las diferencias (Rabello et al., 2006) o de reafirmación étnica desde la recreación de los vínculos comunitarios en el contexto urbano (Planas, 2007). Surge de una diversidad de dispositivos de producción, donde los medios de comunicación de masas vehiculan los discursos hegemónicos (Silverstone, 2004), fomentando la construcción del otro peligroso desde la carga valorativa que éstos le adjudican (Álvarez, 2012). Esta hegemonía no equivale a pasividad, siendo tensionada por dinámicas de valoración de la diferencia, a partir de procesos de resistencia y reafirmación étnica (Merino, Mellor, Sáez, \& Quilaqueo, 2009; Rivera, 2006). 
Como se aprecia desde la revisión presentada, los procesos de subjetivación desde la alteridad permiten establecer distintas siluetas de alteridad, desde la reificación de la peligrosidad, de procesos de hegemonía y resistencia y de naturalización de procesos intersubjetivos, entre otros órdenes posibles. Cabe preguntarse sobre la pertinencia de estos procesos de alteridad, para dar cuenta de la relación entre infancia y delincuencia.

En esta revisión, se observa un grupo de investigaciones que asumen la existencia de jóvenes infractores de ley, en tanto portadores de una particular desviación y/o en una condición de deprivación de los recursos indispensables para su integración. Zambrano y Pérez (2004) proponen que los niños y niñas que cometen infracciones de ley se encuentran condicionados por una serie de factores de carácter psicosocial, situándolos al margen de la integración y evidenciando procesos identitarios de carácter desadaptativo. Valdenegro (2005) plantea que el ejercicio de infracción de ley se encuentra mediado por un conjunto de factores de carácter psicosocial, donde la percepción de ser objeto de prejuicio y el bajo apoyo social familiar son característicos de sujetos con prácticas infractoras. Salazar, Torres, Reynaldos, Figueroa y Valencia (2009) identifican dimensiones de vida en niños infractores desde una perspectiva psicosocial, destacando una asociación significativa entre condiciones de personalidad y la conducta delincuencial. Reyes (2014) enfatiza la especificidad del fenómeno delictivo en adolescentes del género femenino, identificando factores particulares que inciden en su ocurrencia. Morales (2008) revisa las principales teorías explicativas respecto del comportamiento infractor adolescente, enfatizando su carácter multicausal y a la vez resaltando una particular teoría de carácter neuropsicológico (Taxonomía del Desarrollo), con cual presenta el mayor valor heurístico.

Se perfila así un marco investigativo basado en relaciones psicosociales diádicas, individuales y ahistóricas, posibilitándose la emergencia del individuo infractor, relacionado en grados de disfuncionalidad con su entorno de acuerdo a variables individuales y contextuales.
Esta radicación en el individuo y su contexto microsocial pasa por alto las condiciones contextuales macro, omitiendo la producción histórica, cultural y social de la diferencia. En contrapartida, la alteridad en tanto constructo permite situar dicha relación como una expresión cultural, histórica e intersubjetiva, resaltándose la importancia de analizar las condiciones que promueven estas diferenciaciones y a la vez abordar las modalidades específicas donde los dispositivos de producción de alteridad operan en la actualidad.

\section{Relación entre alteridad e infancia desde la noción de dispositivo}

La noción foucaultiana de dispositivo (Agamben, 2011; Deleuze, 1990; Foucault, 2000) denota la idea de "máquinas para hacer ver y hacer hablar" (Deleuze, 1990, p. 155), delineando tanto lo visible y lo invisible, como lo enunciable y lo imposible de ser dicho, haciendo aparecer o desparecer el objeto que define. Así, la problematización de la infancia como sujeto singular en el paradigma garantista es una particular visibilización que desdibuja la existencia de la diversidad de infancias, y específicamente la infancia desde la alteridad.

Según Agamben (2011), dispositivo refiere a un conjunto heterogéneo de elementos tales como discursos, instituciones, edificios, leyes, entre otros. Se aleja de la identificación con la noción de institución, constituyéndose en red de articulación en la medida que responde a un específico orden de inteligibilidad (García, 2011).

La noción de los dispositivos como regímenes (re)productores de órdenes en el mundo permite entenderlos, en tanto líneas de fuerza (Deleuze, 1990), configurando la triple dimensión de saber, poder y subjetividad. Sobre esta última, se les entiende como un proceso sin determinación preexistencial, operando para sujetar al sujeto a un determinado régimen de verdad. Así entendido, la relación entre infancia y delincuencia es contingente cultural e históricamente, delimitada en particulares regímenes de enunciabilidad.

Agamben (2011) señala que los dispositivos producen distintas posiciones de sujeto, pudiendo un 
mismo individuo ser sujeto de múltiples procesos de subjetivación. Se resalta así la importancia central de los dispositivos en los procesos de subjetivación, abriendo la puerta para considerar Lo Otro: "Lo que se subjetiviza son tanto los nobles, (...) así como -aunque en otras condiciones- los excluidos, los malos, los pecadores" (Deleuze, 1990, p. 157).

La construcción de alteridad desde la noción de dispositivo como red articuladora de sentido es posible situarla como proceso de construcción de lo otro en diversos ámbitos del contexto social (espacios comunitarios, medios de comunicación de masas, ámbito jurídico, entre otros), siendo el accionar que el Estado desarrolla en las políticas públicas hacia las infancias uno de los posibles órdenes. No obstante, este ámbito presenta la particularidad de explicitar el vínculo entre infancia y delincuencia desde específicas concreciones tales como planes nacionales y programas de intervención, definiendo conjuntamente las instituciones encargadas de su ejecución, los espacios interventivos y las particulares naturalizaciones sobre a la figura del "infractor de ley".

Así entendido, la política pública y las instituciones hacia las infancias derivadas se constituyen en espacios sociales de construcción de alteridad, sustentados desde saberes y prácticas en torno a la producción de la diferencia (Cabruja, 1998). Se posibilita la reificación de sujetos intervenidos tanto desde modos de actuar como desde saberes, constituyéndose en fábricas de niños (Llobet, 2010), desde su carácter de desviación y peligro.

\section{La producción binaria de infancias como proceso histórico}

Partiendo el enfoque propuesto, la política pública y en particular la generada hacia las infancias se configuran en tanto fenómeno histórico y cultural (Llobet, 2010). Así expuesto, es relevante poder revisar las modalidades en que la acción del Estado hacia las infancias se ha manifestado a través de la historia, intentando comprender tanto las continuidades como las discontinuidades de este ejercicio.

La primera constatación que puede hacerse es que la infancia no es algo dado, erigiéndose en tanto producción social (García \& Gallego, 2011; Gómez -Mendoza \& Alzate-Piedrahíta, 2014; Grupo de Trabajo "Niñez, 2006), la cual dista a su vez de tener una concreción uniforme.

Las infancias se construyen entonces desde específicas relaciones históricas: las redes institucionales, legislaciones, espacialidades y prácticas. De este modo, el Estado y la sociedad civil han materializado históricamente específicos perfiles de infancias, siendo la emergencia de la institución escolar hacia fines del siglo diecinueve un hito histórico en su constitución en Chile. Este proceso se ha traducido en la pedagogización de la infancia (Alzate, 2003; Grupo de Trabajo "Niñez", 2006), decantando el sujeto-niño escolarizado, como la combinación entre objeto de cuidado y de obediencia.

Así entendido, el estatus de niñez se sostiene en torno al comportamiento social deseable, con relativa independencia de cualidades etarias o normativo-garantistas. Se elabora una particular silueta de infancia desde la paradójica negación de su particularidad (Llobet, 2010), lo que decanta como horizonte sobre prácticas cotidianas, restringiendo la pluralidad de las infancias desde un ejercicio dicotómico de producción de lo otro y lo mismo.

Desde una mirada histórica, las acciones del Estado de Chile sobre las infancias dan cuenta de un ejercicio de producción binarista característico de los procesos de construcción de alteridad, en un orden social estatal segregador respecto de determinadas manifestaciones de infancias (Salazar, 2006). La especificidad de la intervención del Estado sobre un segmento de las infancias populares, construye un sujeto cuya desviación es peligrosa, siendo pertinente la modelización de estrategias de control y segregación social. Esta acción de intervención del Estado cobra fuerza desde su carácter sociocultural, heredera de la producción de alteridad étnica del régimen colonial, y como emergente de la interacción cotidiana con lo otro como fuente de ansiedad e inseguridad (Rabello et al., 2006).

El accionar técnico-punitivo se combina con los vaivenes del orden social, en tanto que la infancia popular es objeto de estudio e intervención integracionista desde las ciencia sociales y la técnica social en los ciclos de auge, mientras que en los periodos de 
crisis es objeto de criminalización (Salazar \& Pinto, 2010). Lo anterior no aplica a la subjetivación de infancias en clases sociales media alta y alta, quienes quedan fuera del radio segregador del Estado (Grupo de Trabajo "Niñez", 2006), en donde ser niño-caballerito (Salazar \& Pinto, 2010) propio de la crianza en las capas oligarcas, define un sistema de clase altamente protector y reproductor de las diferencias sociales.

El movimiento reformista de mediados del siglo diecinueve desarrolla espacios sociales de transformación en alianza con el aparato de control social punitivo, configurando desde los sistemas de caridad-control (García, 2001) la figura vulnerable del Menor (Ministerio de Justicia, 1967), como sujeto marginado pero mayoritario. Se cimenta así una cultura de la judicialización de las políticas sociales (Vergara, 2007), construyendo una particular lectura de la infancia, ya en los inicios del siglo pasado (Rocuant, 1932).

Desde los años cincuenta comienza en Chile una expansión de las políticas sociales básicas, facilitándose procesos de integración de amplios sectores sociales, en el contexto del desarrollo del Estado de Bienestar Corporativo (Larrañaga, 2010). Como resultante de este movimiento social ascendente, los menores son cada vez más residuales (García, 2001), conformándose el aparato jurídico como la instancia articuladora de las políticas supletorias de un Estado que concibe a la infancia desde la minoría, en tanto sujeto marginal.

La transformación del Estado que deviene con la dictadura militar acrecienta esta tendencia de judicializar la problemática del menor, aumentando el espacio de intervención desde la aplicación de políticas supletorias, en un trasfondo de carencias materiales. Se desarrolla así una ilusión de política social (García, 2001), reemplazándose el carácter psicosocial y material de la intervención por la figura omnisciente del juez y la normativa jurídica.

En el contexto del proceso de transición a la democracia, los gobiernos de la etapa posdictatorial consolidan una apuesta que conlleva el desarrollo de un nuevo impulso a las políticas sociales en su conjunto, desde el denominado pago de la deuda social (Larrañaga, 2010). Así conceptuado, la infancia pasa a ser un eje de las políticas públicas en diversos frentes, lo que se plasma en la proyección de Planes Nacionales de Infancia, en los periodos 1992-2000, 2001-2010 y 2010-2014 (Gobierno de Chile, 2010).

Estos cuerpos normativos pretenden ser marcos orientadores de la política gubernamental a favor de la infancia y la adolescencia, como respuesta coordinada a la ratificación por parte de Estado de Chile de la Convención sobre los Derechos del Niño (UNICEF, 1989). Se puede sostener que esta redefinición de las políticas públicas desde la valoración de las diferencias sociales y culturales se inscribe en un giro de soporte del nuevo modelo de desarrollo que reemplaza la perspectiva universalista por la de focalización y compensación (Wade, 2006).

No obstante, estos supuestos avances van aparejados de una segmentación institucional, distinguiendo sujetos de protección y sujetos de control, lo que se ha traducido pragmáticamente en la naturalización de grupos sociales desde la criminalización. Así expuesto, se va encarnando la silueta productora de la subjetividad vulnerada, cimentada en la figura del miedo al otro (PNUD, 1998), constituyéndonos desde la diferencia segregadora.

\section{Conclusiones}

Como primera reflexión, es posible sostener la conceptualización crítica de la relación entre infancia y delincuencia desde la noción de alteridad. Lo anterior se enfrenta con la existencia de diversas prácticas naturalizantes que reifican la silueta del joven infractor de ley y definen hegemónicamente la noción del problema social y su abordaje, desde la criminalización hacia las infancias (Sarcinelli, 2011; Sepúlveda, 2011).

A partir de lo anterior, las políticas públicas se constituyen en un importante contexto para la consolidación de la perspectiva normalizadora, conjugando la acción social (integracionista) y la investigación. La psicología social comunitaria permite la visibilización de esta tensión de modo privilegiado, aportando un horizonte crítico y de transformación social desde la promoción de procesos dialógicos entre actores y sus marcos interpretativos (Alfaro, 2013). 
Se desprende así que los programas sociales hacia las infancias se definen como un conjunto de respuestas al particular modelo de desarrollo en Chile, en tanto actualización de las construcciones históricas respecto de la condición y estatus de dichas infancias (Grupo de Trabajo "Niñez", 2006; Salazar, 2006; Rojas, 2010). Es en este sentido que se propone la expresión alter-infancia, connotando la específica subjetivación de otro-sujeto infantil, definido desde las intervenciones sociales hacia las infancias, herederas de la diferenciación históricamente segregadora del Estado-nación chileno. Esta definición de alter-infancia no se traduce en la descripción de un particular modo de ser, resaltándose la condición de proceso en tanto concreción contingente y relativa a dinámicas subjetivadoras inscritas en dispositivos.

A nivel sociocomunitario, concebir la relación entre infancia y delincuencia desde la alteridad se tensiona con los modos de entender la relación entre el mundo adulto y las infancias en un contexto patriarcal y adultocéntrico. La radicalización de los procesos de criminalización expresados en la creciente sensación de inseguridad y en la externalización de los problemas sociales hacia las figuras de alteridad, constituyen un reto para la sociedad en su conjunto, y en particular para las políticas públicas hacia las infancias, si de verdad se proponen situarse desde perspectivas transformadoras y garantistas. En esta óptica, el desarrollo de prácticas de intervención locales orientadas a la promoción de perspectivas garantistas hacia las infancias, en una lógica de desarrollo "abajo-arriba" en que las comunidades se hacen cargo de la resolución y definición de sus problemas, se constituyen en una alternativa no solo necesaria sino eficaz, en contraposición con la ineficaz y verticalista guerra a la delincuencia, en tanto ejemplo de la tendencia privatizadora de los procesos sociales.

Desde esta mirada, los procesos históricos y culturales a la base de la construcción de la alterinfancia se constituyen en un eje de análisis comprensivo que permite problematizar las perspectivas de intervención actuales: seguridad ciudadana, vulnerabilidad, miedo al otro, factores de riesgo, entre otras. Esta constatación, en la medida que nos interpela no solo como investigadores o interventores sociales, sino que en tanto sujetos ético-políticos, permite posicionar un debate necesario respecto de la responsabilidad por el Otro en tanto apuesta histórico-social transformadora (Carballeda, 2013), que polemiza con la naturalización criminalizante que emerge de la reproducción binaria de infancias: niños o jóvenes, víctimas o delincuentes, frágiles o peligrosos.

\section{Referencias}

Agamben, G. (2011). ¿Qué es un dispositivo? Sociológica, 26(73), 249-264. Disponible en http://www.revistasociologica.com.mx/pdf/7310.pdf

Alfaro, J. (2009). Psicología comunitaria y políticas sociales en Chile. Psicología E Sociedade, 21(2), 275- 282.

Alfaro, J. (2013). Psicología comunitaria y políticas sociales: institucionalidad y dinámica de actores. Global Journal of Community Psychology Practice, 4(2), 1-10. Recuperado de http://www.gjcpp.org/ pdfs/alfaro-v4i2-20130613.pdf

Álvarez, M. (2012). De la relación entre identidad y alteridad en las representaciones televisivas de los usuarios de paco. Identidades, 2(2), 42-55. Disponible en https://iidentidadess.files.wordpress. com/2012/06/04-identidades-2-2-2012-alvarezbroz1.pdf

Alzate, M. (2003). La infancia: concepciones y perspectivas. Pereira: Editorial Papiro.

Blaney, L. (2008). Colón y el caníbal. Divergencias. Revista de Estudios Lingüísticos y Literarios, 6(1), 3- 11.

Bernburg, J., Krohn, M., \& Rivera, C. (2006). Official labeling, criminal embeddedness, and subsequent delinquency: A longitudinal test of labeling theory. Journal of Research in Crime and Delinquency, 43(1), 67-88. doi: 10.1177/002242780528006820

Cabruja, T. (1998). Cultura, género y educación: la construcción de la alteridad. En M. Pereyra (Comp.), La educación intercultural en Europa. Un enfoque curricular (pp. 51- 66). Barcelona: Pomares-Corredor.

Carballeda, A. (2013). La intervención en lo social desde una perspectiva americana. Algunos aportes de Enrique Dusell y Rodolfo Kusch. Margen, 70, 
1-6. Recuperado de http://www.margen.org/suscri/ margen70/carballeda.pdf

Castro-Gómez, S. (2000). Ciencias sociales, violencia epistémica y el problema de la 'invención' del otro. En E. Lander (Comp.), La colonialidad del saber: eurocentrismo y ciencias sociales. Perspectivas latinoamericanas (pp. 145-161). Buenos Aires: CLACSO.

Cole, K., Mills, P., Jenkins, J., \& Dale, P. (2005). Early intervention curricula and subsequent adolescence social development: A longitudinal examination. Journal of Early Intervention, 27(2), 71- 82.

Colmenares, Y. (2004). La otredad clausurada: prácticas escolares para la mismidad. Heterotopía, 9(27), 45-59.

Crooks, C., Scott, K., Ellis, W., \& Wolfe, D. (2011). Impact of a universal school- based violence prevention program on violent delinquency: Distinctive benefits for youth with maltreatment histories. Child Abuse and Neglect, 35(6), 393- 400.

Deleuze, G. (1990). ¿Qué es un dispositivo? En G. Deleuze (Coord.), Michel Foucault filósofo (pp. 155163). Barcelona: Gedisa.

Flórez, J. (2007). Lectura no eurocéntrica de los movimientos sociales. Las claves analíticas del proyecto modernidad/colonialidad. En J. Castro-Gómez \& R. Grosfoguel (Eds.), El giro decolonial. Reflexiones para una diversidad epistémica más allá del capitalismo global (pp. 243 - 266). Bogotá: Siglo del Hombre Editores. Disponible en http://www.unsa.edu. ar/histocat/hamoderna/grosfoguelcastrogomez.pdf

Fondo de las Naciones Unidas para la Infancia (1989). Convención Internacional sobre los Derechos del Niño. Santiago: Autor.

Fondo de las Naciones Unidas para la Infancia.(2008). Principales nudos problemáticos de los Centros Privativos de Libertad para Adolescentes y Secciones Penales Juveniles (Informe Ejecutivo). Santiago: Autor.

Foucault, M. (1991). Tecnologías del yo. Barcelona: Paidós.

Foucault, M. (2011). Los anormales. Buenos Aires: Fondo de Cultura Económica.

Galvis, L. (2009). La Convención de los Derechos del Niño veinte años después. Revista Latinoamericana de Ciencias Sociales, Niñez y Juventud, 7(2), 587-619.

García, E. (2001). Prehistoria e historia del control sociopenal de la infancia: política jurídica y derechos humanos en América Latina. En S. Larraín \& D.
Mettifogo (Comps.), Materiales para el estudio y la intervención en infancia (pp. 25- 33). Santiago de Chile: Universidad de Chile.

García, F. (2011). ¿Qué es un dispositivo?: Foucault, Deleuze, Agamben. A Parte Rei. Revista de Filosofía, 74, 1-8. Recuperado de http://serbal.pntic.mec. es/ cmunoz11/fanlo74.pdf

García, G., \& Gallego, T. (2011). Una concepción abierta e interdisciplinaria de la infancia. Revista Latinoamericana de Ciencias Sociales, Niñez y Juventud, 9(2), 17- 25.

Gatti, U., Tremblay, R., \& Vitaro, F. (2009). Iatrogenic effect of juvenile justice. The Journal of Child Psychology and Psychiatry, 50(8), 991- 998.

Gnecco, C. (2008). Discursos sobre el otro: pasos hacia la arqueología de la alteridad étnica. Revista CS de Ciencias Sociales, 2, 101-129.

Gobierno de Chile (2010). Política nacional y plan de acción integrado en favor de la infancia y la adolescencia 2010-2014. Santiago de Chile: Ministerio de Desarrollo Social.

Gómez-Mendoza, M., \& Alzate-Piedrahíta, M. (2014). La infancia contemporánea. Revista Latinoamericana de Ciencias Sociales, Niñez y Juventud, 12(1), 77-89.

Greenwood, P. (2008). Prevention and intervention programs for juvenile offenders. The Future of Children, 18(2), 185-210.

Grupo de Trabajo "Niñez".(2006). Conceptualizaciones de la niñez desde la lectura de las políticas públicas en infancia. Revista de Psicología, 15(2), 55-63. http://www.revistapsicologia.uchile.cl/index.php/ RDP/article/viewFile/18396/19433

González, F. (2009). Alteridad en estudiantes. Entre la alteración y el equilibrio. Revista Mexicana de Investigación Educativa, 14(42), 889- 910.

Hawkins, J., Kosterman, R., Catalano, R., Hill, K., \& Abbot, R. (2008). Effects of social development intervention in childhood fifteen years later. Archives of Pediatric $\mathbb{E}$ Adolescent Medicine, 162(12), 1133-1141. doi: 10.1001/archpedi.162.12.1133

Heffes, A. (2012). La identidad revisitada. Identidades, 3, 85- 97.

Herrera, J. (2006). Colonialismo y violencia. Bases para una reflexión pos-colonial desde los derechos 
humanos. Revista Crítica de Ciências Sociais, 75, 21-40.

Instituto Nacional de Derechos Humanos (2013). Situación de los derechos humanos en Chile (Informe anual 2013). Santiago de Chile: Autor.

Jenson, J. (2010). Advances in preventing childhood and adolescent problem behavior. Research on Social Work Practice, 20(6), 701-713.

Juliano, D. (1994). La construcción de la diferencia: los latinoamericanos. Papers, 43, 23- 32.

Larrañaga, O. (agosto, 2010). Las nuevas políticas de protección social en perspectiva histórica (Documento de trabajo). Santiago: PNUD.

Llobet, V. (2010). iFábrica de niños? Las instituciones en la era de los derechos de la infancia. Buenos Aires: Editorial Noveduc.

Mann, E., \& Reynolds, A. (2006). Early intervention and juvenile delinquency prevention: Evidence from the Chicago longitudinal study. Social Work Research, 30(3), 153-167.

Merino, M., Mellor, D., Saiz, J., \& Quilaqueo, D. (2009). Perceived discrimination amongs indigenous people in Chile: An application of the Australian taxonomy. Journal of Ethnic and Racial Studies, 32(5), 802-822.

Ministerio de Justicia. Ley 16.618 de 1967. Fija el texto definitivo de la Ley de Menores. Marzo 8 de 1967.

Ministerio de Justicia. Ley 20.084 de 2005. Establece un sistema de responsabilidad de los adolescentes por infracciones a la ley penal. Diciembre 7 de 2005.

Montenegro, M., \& Pujol, J. (2003). Conocimiento situado: un forcejeo entre el relativismo construccionista y la necesidad de fundamentar la acción. Revista Interamericana de Psicología, 37(2), 295-307.

Montero, M. (2010). Crítica, autocrítica y construcción de teoría en la psicología social latinoamericana. Revista Colombiana de Psicología, 19(2), 177-191.

Montero, M. (2011). Nuevas perspectivas en psicología comunitaria y psicología social crítica. Ciencias Psicológicas, 5(1), 61-68.

Montero, M., \& Montenegro, M. (2006). Critical psychology in Venezuela. Anual Review of Critical Psychology, 5, 257-268.

Morales, H. (2008). Factores asociados y trayectorias del desarrollo del comportamiento antisocial durante la adolescencia: implicancias para la prevención de la violencia juvenil en América Latina. Revista Interamericana de Psicología, 42(1), 129-142.

Olmos, A. (2012). Cuando migrar se convierte en estigma: un estudio sobre construcción de alteridad hacia la población inmigrante extranjera en la escuela. Imagonautas, 1(2), 62-85.

Piola, M. (2008). Alteridad y cultura: "Ninguna mujer nace para puta". Kairos. Revista de Temas Sociales, 21, 1-19. Disponible en http://www.revistakairos. org $/ \mathrm{k} 21-02 . \mathrm{htm}$

Piper, I. (2002). Sobre una práctica que, en el sur, se llama a sí misma psicología social crítica. En I. Piper (Ed.), Políticas, sujetos y resistencias: debates y críticas en psicología social. Cuadernos de Psicología Social (pp. 19-31). Santiago de Chile: ARCIS.

Planas, M. (2007). Identidad étnica en el Perú: un estudio cualitativo sobre los discursos de autoidentificación en tres zonas del país. Lima: Universidad Particular Cayetano Heredia.

Programa de las Naciones Unidas para el Desarrollo (1998). El miedo al otro: la seguridad ciudadana. Santiago: ONU.

Quiroz, A. (2011). Veinte años de la Convención de los Derechos del Niño: reflexiones sobre el desarrollo jurídico en Colombia. Revista Criterio Jurídico Garantista, 5, 42-50.

Rabello, L., Rocha, A., Texeira, E., Alves, R., \& Antunes, H. (2006). La construcción de la diferencia: la juventud en la ciudad y sus relaciones. Psicologia em Estudo, 11(2), 437-447. Disponible en http:// www.scielo.br/pdf/pe/v11n2/v11n2a22.pdf

Rea, A. (2006). La europeización de la política migratoria y la transformación de la otredad. Revista Española de Investigaciones Sociológicas, 116, 157-184.

Recasens, A. (2006). Aproximaciones antropológicas al fenómeno de la violencia. Revista de Antropología, $18,31-58$.

Reyes, C. (2014). ¿Por qué las adolescentes chilenas delinquen? Política Criminal, 9(17), 1-26.

Reynolds, A., Ou, S., \& Topitzes, J. (2004). Paths of effects of early childhood intervention on education attainment and delinquency: A confirmatory analysis of the Chicago Child- Parent Centers. Child Development, 75(5), 1299-1328. 
Rivera, F. (2006). Entorno neoliberal y la alteridad étnica antiflexible de los atacameños contemporáneos. Revista de Antropología, 18, 59-89.

Rocuant, J. (1932). La delincuencia infantil y los reformatorios de niños. Santiago: La Tarde.

Rojas, J. (2010). Historia de la infancia en el Chile republicano, 1810, 2010. Santiago: JUNJI.

Salazar, G. (2006). Ser niño "huacho" en la historia de Chile (siglo XX). Santiago: LOM.

Salazar, G., \& Pinto, J. (2010). Historia contemporánea de Chile V. Santiago: LOM.

Salazar, J., Torres, T., Reynaldos, C., Figueroa, N., \& Valencia, S. (2009). Perspectiva psicosocial en adolescentes acusados por delitos en contra de la propiedad. Revista Latinoamericana de Ciencias Sociales, Niñez y Juventud, 7(2), 1491-1512.

Sarcinelli, A. (2011). Infancias marginales, los márgenes de la infancia. Trayectorias de muchachos en situación de calle en el noreste brasileño. Alteridades, 21(42), 91-101.

Sepúlveda, R. (diciembre, 2011). Imaginarios de la niñez en el contexto de las reformas penales en Chile y Argentina. Ponencia presentada en el X Congreso Argentino de Antropología Social, Universidad de Buenos Aires, Argentina.

Servicio Nacional para la Prevención y Rehabilitación del Consumo de Drogas y Alcohol (2013). Construyendo culturas preventivas: guía para el abordaje del consumo y tráfico de drogas y alcohol desde las comunidades educativas. Santiago de Chile: Ministerio del Interior y Seguridad Pública.
Silverstone, R. (2004). ¿Por qué estudiar los medios? Buenos Aires: Amorrortu.

Smith, R. (2009). Childhood, agency and youth justice. Children E⿱ Society, 23(4), 252-264.

Soria, S. (2012). Diferencia cultural, tiempo y espacio: notas de y para una investigación en torno a la alteridad indígena. Identidades, 2, 1-23.

Trentacosta, C., \& Shaw, D. (2009). Emotional selfregulation, peer rejection, and antisocial behavior: Developmental associations from early Childhood to early adolescence. Journal of Applied Developmental Psychology, 30(3), 356-365.

Valdenegro, B. (2005). Factores psicosociales asociados a la delincuencia juvenil. Psykhe, 14(2), 33-42.

Vergara, A. (2007). La intervención social como conflicto. El caso de la infancia y juventud en Chile. Revista Praxis, 9(11), 127-131.

Wade, P. (2006). Etnicidad, multiculturalismo y políticas sociales en Latinoamérica: poblaciones afrolatinas e indígenas. Tabula Rasa, 4, 59-81.

Werth, F. (2010). Protección de derechos y responsabilidad penal adolescente: el desafío de la nueva institucionalidad. Santiago de Chile: Paz Ciudadana.

White, B., Temple, J., \& Reynolds, A. (2010). Predicting adult criminal behavior from juvenile delinquency: Ex ante and ex post benefits of early Intervention. Advances in Life Course Research, 15(4), 161-170.

Zambrano, A., \& Pérez, R. (2004). Construcción de identidad en jóvenes infractores de ley, una mirada desde la psicología cultural. Revista de Psicología, 13(1), 115-132. 
